\title{
Perspectives of Gynecologic Oncologists on Minimally Invasive Surgery During COVID-19 Pandemic: A Turkish Society of Minimally Invasive Gynecologic Oncology (MIJOD) Survey
}

\author{
Nasuh Utku Dogan ${ }^{1 *}$, Esra Bilir ${ }^{2}$, Salih Taskin ${ }^{3}$, Dogan Vatansever ${ }^{2}$, Selen Dogan ${ }^{1}$, \\ Cagatay Taskiran', Husnü Celik ${ }^{4}$, Firat Ortac ${ }^{3}$, Mete Gungor ${ }^{5}$
}

\begin{abstract}
Background: To evaluate gynecologic oncologists' trends and attitudes towards the use of Minimally invasive surgery (MIS) in active period of the COVID-19 pandemic in Turkey. Methods: Online national survey sent to members of Turkish Endoscopy Platform consisting of six sections and 45 questions between the dates 1-15 June 2020 in Turkey to explore their surgical practice during the pandemic in three hospital types: Education and research hospital/ university hospital, state hospital and private Hospital. Participants were gynecologic oncologists who are members of Turkish Endoscopy Platform. Results: Fifty-eight percent of participants canceled all operations except for cancer surgeries and emergent operations. About a quarter of participants (28\%) continued to operate laparoscopically and/or robotically. For the evaluation of the suspected adnexial mass (SAM) 64\% used laparotomy and only $13 \%$ operated by laparoscopy $(\mathrm{L} / \mathrm{S})$. For the management of low-risk early-stage endometrial cancer only fifth of the participants preferred to perform L/S. For endometrial cancer with high-intermediate risk factors more than half of participants preferred complete staging with laparotomy. For advanced stage ovarian cancer, one-fifth of the participants preferred to perform an explorative laparotomy, whilst $15 \%$ preferred diagnostic laparoscopy to triage the patients for either NACT or cytoreductive surgery. On the contrary $41 \%$ of participants chose to have cytology by paracentesis for neo-adjuvant chemotherapy (NACT). Gynecologic oncologists with $>10$ years L/S experience used MIS more for SAM. Furthermore, experienced surgeons used L/S more for endometrial cancer patients. In busy COVID hospitals, more participants preferred laparotomy over L/S. Conclusion: Use of MIS decreased during the pandemic in Turkey. More experienced surgeons continued to perform MIS. Surgical treatment was the preferred approach for SAM, early-stage endometrial cancer. However, NACT was more popular compared to radical surgery.
\end{abstract}

Keywords: COVID-19- gynecologic oncology- survey- laparoscopy- Turkey

Asian Pac J Cancer Prev, 23 (2), 573-581

\section{Introduction}

After the emergence of severe acute respiratory syndrome coronavirus 2 (SARS-CoV-2) infection in China and quick transmission of the disease worldwide, routine practices in all fields of clinical medicine have changed significantly (Nakayama et al., 2020; Soreide et al., 2020). This situation affected the way we examine the patients, the way we operate, and even the way we communicate with each other. Particularly in countries where a high number of infected patients obstructed the medical infrastructure, all routine patient care and operations were either canceled or postponed (Spinelli and Pellino, 2020).

When the first case in Turkey was announced on11
March 2020, exactly the same date as World Health Organization (WHO) declared Coronavirus disease 2019 (COVID-19) as a pandemic, specific regulations were introduced by Turkish Ministry of Health including cancellation of elective benign surgeries, reserving and restricting intensive care unit beds only to patients with COVID-19, and assigning the healthcare professionals to COVID-19 wards where more resources were needed (Cucinotta and Vanelli, 2020; Demirbilek et al., 2020).

In parallel with the publication of position statement by international societies, Turkish Society of Gynecologic Oncology (TRSGO) and Turkish Society of Minimally Invasive Gynecologic Oncology (MİJD) also launched their recommendations regarding the care of patients with

${ }^{1}$ Department of Gynecology, Akdeniz University Hospital, 07070, Antalya, Turkey. ${ }^{2}$ Department of Gynecology, Koc University Hospital, 34010, Zeytinburnu/Istanbul, Turkey. ${ }^{3}$ Department of Gynecology, Ankara University Hospital, 06100, Mamak/ Ankara, Turkey. ${ }^{4}$ Department of Gynecology, Baskent University Adana Hospital, 01250, Yuregir/Adana, Turkey. ${ }^{5}$ Department of Gynecology, Aclbadem Mehmet Ali Aydinlar University, 34457, Maslak/Istanbul, Turkey. *For Correspondence: nasuhutkudogan@yahoo.com 
gynecologic malignancies and oncologic surgery during the pandemic(Fader et al., 2020; Oncology, 2020; Uwins et al., 2020; (MİJOD), 2020). In addition to postponing elective benign surgeries, all these societies recommended classifying and assigning patients with gynecologic malignancies into different groups with respect to the urgency of their condition(Fader et al., 2020; Rusch et al., 2020; Uwins et al., 2020). Minimally invasive surgery (MIS), including laparoscopy and robotic surgery, has replaced open procedures in many fields of gynecologic oncologic practices, which all improves the quality of life of patients, enhances faster recovery, and shortens the length of hospitalization (Kornblith et al., 2009). However, in the period of COVID-19 pandemic because of the uncertainties regarding the possible transmission of the virus with the use of newer energy modalities, the risk of carbon dioxide $\left(\mathrm{CO}_{2}\right)$ leakage from trocars, there is a significant refrain from laparoscopic surgery (Di Saverio et al., 2020). Moreover, anecdotal studies reported a higher virus load on the peritoneal cavity, which all contributed to a decreased use of MIS in gynecologic oncologic practice (Coccolini F, 2020) Although the societies have quickly published their guidelines amid the pandemic, the real-life experience regarding the use of MIS for the management of patients with gynecologic malignancies has not been fully investigated. In this cross-sectional nationwide study, we aimed to evaluate gynecologic oncologists' trends and attitudes towards the use of MIS in the active period of the COVID-19 pandemic in Turkey.

\section{Materials and Methods}

This cross-sectional survey study was conducted between the dates 1-15 June 2020 in Turkey, where the restrictions related to COVID-19 pandemic were released on 1st June 2020. The Turkish Ministry of Health (2020-06-03T09-58-03) and Koc University Institutional Review Board approved our study (2020.260.IRB3.101). Our inclusion criteria included i) actively involving in the surgical management of gynecologic cancers, including gynecologists, gynecologic oncology fellow in training, and certified gynecologic oncologist, ii) practicing in Turkey, and iii) completing the survey. Before the official launch of the survey, linguistic and grammatical consistency were validated with 32 voluntary ob\&gyn residents and these data were not included for the final data analysis.

An online informed consent was obtained from the participants in order to proceed to the survey. We did not collect any identifiable information and hence the anonymity of the participants was not violated. The survey was distributed to the members of Turkish Society of Minimally Invasive Gynecologic Oncology (MIJOD). The members were also encouraged to distribute the survey to their colleagues, meeting our inclusion criteria. The survey link was shared through MIJOD's social media accounts, such as Instagram and Twitter. The sample size was calculated as 98 where population size, confidence interval, and margin of error were 130 (current number of the MIJOD group members), 95\%, and 5\%, respectively. We decided to inactivate the survey link upon reaching out to our sample size. A total number of 135 people filled in our survey during the study period. We excluded eight, ten, and seventeen participants because they did not proceed to the survey upon giving IC, met the exclusion question, and did not complete the survey, respectively. A total of 100 out of 135 participants met the inclusion criteria, where we met our pre-calculated sample size and were included for the final analysis. Given the population size of 130, the response rate was $76.9 \%$. The median duration to complete the survey was 10.85 minutes (range 7.9-16.4 min).

The survey was prepared by Qualtrics in Turkish Language (http://koc.ca1.qualtrics.com/jfe/form/ SV 3ypxCU17cB6WaKF). The Qualtrics calculated the estimated time for the survey completion as 13.3 minutes. The survey comprised of six sections and 45 questions. Immediately after obtaining informed consent referred to as the first section to proceed to the survey, we asked an exclusion question to the participants' involvement in the surgical management of the patients with gynecologic malignancies, referred to as the second section. If they chose "No," the survey was finished without showing any further questions.

In the third section, the demographic characteristics of the participants were asked including age, sex, academic title, years of experience in gynecologic oncology, type of the institution practicing at, the region of the practice, years of experience in laparoscopic surgery $(\mathrm{L} / \mathrm{S})$ and robotic surgery (RS), and years of experience in $\mathrm{L} / \mathrm{S}$ and RS for the management of the gynecologic cancers. We also investigated their history of COVID-19 testing, COVID-19 prophylaxis such as chloroquine, COVID-19 diagnosis, and COVID-19 treatment. Since the pandemic has been devastating for healthcare workers, we included questions to understand their emotional status changes and concerns.

The fourth section was prepared to explore the changes in their working environment, their patients' COVID-19 status, postoperative mortality due to COVID-19, their surgical practice changes during the pandemic, their thoughts about the MIS concerning the SARS-CoV-2 infection, and their practice about obtaining additional informed consent (IC) including SARS-CoV-2 related risks. We aimed to search their attitudes regarding the diagnostic tests for the gynecologic malignancies, such as endometrial biopsy, fractional curettage, colposcopy and/ or cervical biopsy, loop electrosurgical excision procedure (LEEP) or conization, and hysteroscopy. Although our primary goal was to investigate the management of the gynecologic malignancy during the pandemic, one question was added to the survey to check their practice on the benign gynecologic cases during the pandemic.

We included questions regarding the pre-operative COVID-19 testing strategies, types of personal protective equipment (PPE) used during the surgery, and the precautions taken during MIS to the fifth section. We specifically added a question to investigate their preference for ultrasonic energy modality during $\mathrm{L} / \mathrm{S}$ due to the anecdotal studies reporting a high number of viral particles in smokes generated by ultrasonic energy devices(Givi et al., 2020).

The final section was created to explore their surgical 
practice during the pandemic. Their approach to the management of the cases with suspected adnexal mass (SAM) and when started the surgery as MIS, if the diagnosis of ovarian cancer was made intra-operatively on cases with SAM, were questioned. We asked questions to reveal their management of the early and advanced stages of ovarian cancer, endometrial cancer, and cervical cancer during the pandemic.

In order to understand different parameters on practices in pandemic, the whole study population was divided in to two groups with respect to age (older or younger than 40 years of age) and experience in minimally invasive surgery (less than ten years or more) and experience in gynecologic oncologic surgery (less than five years or more)

\section{Statistics}

Statistical Package for Social Sciences (SPSS) Version 26.0 (Chicago, IL, USA) was used to analyze the data. We used the SPSS file automatically generated by Qualtrics to minimize the errors caused by manual data entrance. The normality of each one of our variables was evaluated with Kolmogorov-Smirnov and Shapiro-Wilk tests, where the data was accepted as normally distributed if the alpha value is $>0.05$. We performed the statistical analysis to calculate mean values ( \pm standard deviation) for normally distributed data, median values (interquartile, $25^{\text {th }}-75^{\text {th }}$ percentile) for non- normally distributed data, and percentages. The pairwise comparisons between two independent groups were evaluated with Mann-Whitney U test. Chi-square and Fisher's exact tests were used for categorical data. $\mathrm{P}<0.05$ was assigned to be statistically significant.

\section{Results}

Median age was 40 years (range 30-66) (Table 1). The majority of the participants $(82.0 \%)$ were male. Almost one-fifth of the participants $(18 \%)$ were gynecologic oncology fellows. More than half of them $(64 \%)$ were working in a university hospital, while a quarter of participants was working in a state hospital. $82 \%$ of the participants was practicing L/S for more than five years. Almost one-third of the participants $(27.0 \%)$ were evaluated for COVID-19 infection, where $16 \%$ received any kind of prophylaxis. Only one physician (1.0\%) was diagnosed with COVID-19 infection and was not hospitalized but instead had a home quarantine. Half of the participants expressed feelings of anxiety and fear of getting SARS-CoV-2 infection during both MIS and open surgery. About $40 \%$ of participants working in a hospital designated as low work-load with respect to COVID-19 the pandemic, and they expressed that they had enough resources for on-going surgical procedures (Table 2). However, one-third of the participants working in a pandemic hospital with a high patient load in which routine surgical practice was restricted. Regarding the regular educational meetings and tumor boards, nearly half of the participants (47\%) continued these activities by telemedicine (video-conference). Eleven patients (2.7 $\%)$ were diagnosed with SARS-CoV-2 infection through
Table 1. Baseline Characteristics of the Participants

\begin{tabular}{|c|c|}
\hline Variable $(\mathrm{n}=100)$ & $\begin{array}{c}\text { Number } \\
(\%)\end{array}$ \\
\hline \multicolumn{2}{|l|}{ Exclusion Question } \\
\hline Gynecologic Oncologist & 62 \\
\hline Fellow & 18 \\
\hline Involves in MoGC & 20 \\
\hline Age in years & 40 \\
\hline (range) & $(30-66)$ \\
\hline \multicolumn{2}{|l|}{ Sex } \\
\hline Female & 18 \\
\hline Male & 82 \\
\hline \multicolumn{2}{|l|}{ Institution } \\
\hline Education and Research Hospital & 23 \\
\hline Foundation University Hospital & 8 \\
\hline Private Hospital & 10 \\
\hline State Hospital & 3 \\
\hline University Hospital & 56 \\
\hline \multicolumn{2}{|l|}{ Title } \\
\hline Fellow & 18 \\
\hline Gynecologic Oncologist & 18 \\
\hline Assistant Professor Dr & 8 \\
\hline Associate Professor Dr & 27 \\
\hline Professor Dr & 24 \\
\hline Other & 5 \\
\hline \multicolumn{2}{|l|}{ Experience years (mean) } \\
\hline Gynecologic oncology & 9 \\
\hline $\mathrm{L} / \mathrm{S}$ & 10 \\
\hline $\mathrm{L} / \mathrm{S}$ in MoGC & 5.9 \\
\hline RS & 17.3 \\
\hline RS in MoGC & 17.1 \\
\hline \multicolumn{2}{|l|}{ COVID-19 Testing } \\
\hline Yes & 27 \\
\hline No & 73 \\
\hline \multicolumn{2}{|l|}{ COVID-19 Prophylaxis } \\
\hline Yes & 16 \\
\hline No & 84 \\
\hline \multicolumn{2}{|l|}{ COVID-19 Diagnosis } \\
\hline Yes & 1 \\
\hline No & 99 \\
\hline \multicolumn{2}{|l|}{ COVID-19 Treatment* } \\
\hline Yes, isolation & 1 \\
\hline Yes, hospitalization & - \\
\hline No & 99 \\
\hline \multicolumn{2}{|l|}{ Emotional Status Changes* } \\
\hline Yes, anxious about infecting with SARS-CoV2 & 53 \\
\hline Yes, anxious about infecting relatives with SARS-CoV2 & 72 \\
\hline No & 11 \\
\hline \multicolumn{2}{|l|}{ Concern during Surgery* } \\
\hline Only L/S and RS & 27 \\
\hline Laparotomy & 58 \\
\hline None & 30 \\
\hline
\end{tabular}

*Multiple choices were allowed to be selected; L/S, laparoscopic surgery; MoGC, management of gynecologic cancers; SARS-CoV 2, Severe Acute Respiratory Syndrome Coronavirus 2; Robotic Surgery, RS

Asian Pacific Journal of Cancer Prevention, Vol 23 
Table 2. Impact of COVID-19 on Surgical Practice

\begin{tabular}{|c|c|}
\hline Variable & $\begin{array}{c}\text { Number } \\
(\%)\end{array}$ \\
\hline \multicolumn{2}{|l|}{ Institution } \\
\hline No COVID-19 & 9 \\
\hline Not many COVID-19 cases, enough ICU and OR & 39 \\
\hline Many COVID-19 cases and inadequate ICU and OR & 30 \\
\hline Many COVID-19 cases, but adequate ICU and OR & 16 \\
\hline ICU and OR exclusive for COVID-19 & 5 \\
\hline Other & 1 \\
\hline \multicolumn{2}{|l|}{ Educational Activities } \\
\hline Not affected & 1 \\
\hline Decreased & 11 \\
\hline Virtually continued & 47 \\
\hline All canceled & 38 \\
\hline Other & 3 \\
\hline \multicolumn{2}{|l|}{ Surgery } \\
\hline COVID-19 positive cases, detected by CT or PCR & 11 \\
\hline $\begin{array}{l}\text { COVID-19 suspected cases, not detected by CT or PCR } \\
\text { and symptoms }\end{array}$ & 66 \\
\hline $\begin{array}{l}\text { COVID- } 19 \text { negative cases, not detected by CT or PCR } \\
\text { and no symptoms }\end{array}$ & 322 \\
\hline \multicolumn{2}{|l|}{ PO COVID-19 positivity } \\
\hline first 7 days & 3 \\
\hline first 14 days & 1 \\
\hline first 30 days & 1 \\
\hline Mortality perioperative due to COVID-19 & 2 \\
\hline \multicolumn{2}{|l|}{ Change in Surgical Practice } \\
\hline All the surgeries postponed & 9 \\
\hline $\begin{array}{l}\text { All the surgeries canceled, and the patients referred to } \\
\text { other centers }\end{array}$ & 3 \\
\hline Except emergencies, all the surgeries postponed & 15 \\
\hline $\begin{array}{l}\text { Except cancer and emergencies, all the surgeries } \\
\text { postponed }\end{array}$ & 58 \\
\hline $\begin{array}{l}\text { Except cancer, emergencies, and selected benign, all the } \\
\text { surgeries postponed }\end{array}$ & 10 \\
\hline Other & 2 \\
\hline \multicolumn{2}{|l|}{ Does MIS have more COVID-19 infectivity compared to L/T? } \\
\hline Yes & 58 \\
\hline No & 42 \\
\hline \multicolumn{2}{|l|}{ Continue to MIS } \\
\hline Yes & 28 \\
\hline Only for selected cases & 29 \\
\hline No & 43 \\
\hline \multicolumn{2}{|l|}{ Reasons to discontinue MIS* } \\
\hline Not discontinued & 30 \\
\hline Risk of infection & 41 \\
\hline Society guidelines & 18 \\
\hline Inadequate PPE & 6 \\
\hline Inadequate $\mathrm{OR}$ conditions & 27 \\
\hline Other & 9 \\
\hline \multicolumn{2}{|l|}{ IC specific for COVID-19 infection } \\
\hline Yes & 76 \\
\hline No & 24 \\
\hline \multicolumn{2}{|l|}{ Surgery for Benign Cases } \\
\hline Yes, only $\mathrm{L} / \mathrm{T}$ or vaginally & 10 \\
\hline Yes, only MIS & 4 \\
\hline
\end{tabular}

Table 2. Continued

\begin{tabular}{lc}
\hline Variable & $\begin{array}{c}\text { Number } \\
(\%)\end{array}$ \\
\hline Surgery for Benign Cases & \\
No & 82 \\
Other & 4 \\
\hline
\end{tabular}

IC, informed consent; ICU, intensive care unit; L/T, laparotomy; MIS, minimally invasive surgery; OR, operating room; PO, postoperative; PPE, personal protective equipment; * Multiple choices were allowed to be selected

polymerase chain reaction (PCR) or thorax computed tomography $(\mathrm{CT})$ while 66 symptomatic patients (16.5 $\%$ ) without any detectable findings in PCR or thorax CT underwent an operation. In total, 322 patients were operated who had neither symptoms nor any detected positive results for SARS-CoV-2 infection. Two patients $(0.5 \%)$ died of COVD-19 during the per-operative period. Five patients $(1.0 \%)$ were diagnosed with SARS-CoV-2 infection during the post-operative period, three on postoperative day seven and two within the first post-operative month. More than half of the participants $(58 \%)$ canceled all the operations except for cancer surgeries and emergent operations. About a quarter of participants (28\%) continued to operate laparoscopically and/or robotically. About third of the participants $(29 \%)$ used MIS only in selected patients. Forty percent of the participants discontinued MIS with a fear of increased infectious contamination risk. Concerning diagnostic procedures, such as endometrial biopsy, LEEP, colposcopy, and cervical biopsy either in the outpatient clinics or under general anesthesia, most of the participants $(75 \%)$ expressed that they continued to do these procedures except colposcopy, cervical biopsy, and LEEP which were all postponed by the participants to a later time (Table 3). Benign gynecologic cases were either canceled or postponed to another time period by $82 \%$ of the participants.

In order to screen COVID-19 preoperatively in patients with a plan of MIS, more than half of the participants (57\%) only checked the COVID-19 symptoms, a third (38\%) took nasopharyngeal swabs for PCR, and a fifth of the participants $(21 \%)$ ordered thorax CT (Table 4$)$.

Regarding the routine PPE use during MIS, nearly a

Table 3. Strategy for Diagnostic Surgical Interventions during COVID-19 Pandemi

\begin{tabular}{lcc}
\hline Variable & $\begin{array}{c}\text { Continued } \\
(\%)\end{array}$ & $\begin{array}{c}\text { Discontinued } \\
(\%)\end{array}$ \\
\hline Endometrial Biopsy at OC & 75 & 25 \\
Endometrial Biopsy under GA & 53 & 47 \\
Fractional DC at OC & 67 & 33 \\
Fractional DC under GA & 49 & 51 \\
Colposcopy and/or Cervical Biopsy at OC & 70 & 30 \\
Colposcopy and/or Cervical Biopsy under & 34 & 66 \\
GA & & \\
LEEP or conization at OC & 41 & 59 \\
LEEP or conization under GA & 43 & 57 \\
Hysteroscopy & 24 & 76 \\
\hline
\end{tabular}

DC, dilation and curettage; GA, general anesthesia; LEEP, Loop electrosurgical excision procedure; $\mathrm{OC}$, outpatient clinic. 
third (29\%) used two surgical masks, another third (37\%) used face masks, and fifth (20\%) used FFP3. Six percent of the gynecologic oncologists discontinued ultrasonic energy use, whereas $15 \%$ of the participants used it routinely as before, and another $15 \%$ only decreased energy use.

For the evaluation of the SAM during the pandemic, a quarter of the participants postponed surgery at a later time, while more than half (64\%) used laparotomy and 13 $\%$ operated by L/S. If the laparoscopically operated SAM cases were found out to be malignant, nearly three-quarters of the participants $(73 \%)$ converted to laparotomy as the

Table 4. Precautions for MIS during the Pandemic

\begin{tabular}{|c|c|}
\hline Variable & Number $(\%)$ \\
\hline \multicolumn{2}{|l|}{ Pre-operative* } \\
\hline Symptom questioning & 57 \\
\hline PCR with nasopharyngeal swab & 38 \\
\hline Routine thorax CT & 21 \\
\hline PPE & 23 \\
\hline No MIS & 32 \\
\hline Other & 4 \\
\hline \multicolumn{2}{|l|}{ PPE* } \\
\hline Astronaut Cap & 9 \\
\hline Single Surgical Masks & 37 \\
\hline Two Surgical Mask & 29 \\
\hline Face Shield & 32 \\
\hline FFP3 & 20 \\
\hline N95 & 38 \\
\hline Boot & 4 \\
\hline Other & 5 \\
\hline \multicolumn{2}{|l|}{ Surgery* } \\
\hline Negative OR Pressure & 10 \\
\hline HEPA filter & 8 \\
\hline ULPA filter & 2 \\
\hline Low intraabdominal pressure & 29 \\
\hline Surgical Smoke Filter & 6 \\
\hline Closed suction system & 13 \\
\hline Balloon Trocar & 3 \\
\hline Less in and out movement of the Trocars & 21 \\
\hline Using the energy modalities less frequently & 11 \\
\hline No precautions & 29 \\
\hline No MIS & 38 \\
\hline Other & 7 \\
\hline \multicolumn{2}{|l|}{ Ultrasonic Energy* } \\
\hline Not used before the pandemic & 21 \\
\hline Quitted & 6 \\
\hline Less frequently used & 15 \\
\hline Continue to use liberally & 15 \\
\hline No MIS & 43 \\
\hline
\end{tabular}

CT, Computed tomography; FFP, filtering facepiece; HEPA, high efficiency particulate air; MIS, minimally invasive surgery; OR, operating room; PCR, Polymerase chain reaction; PPE, personal protective equipment; ULPA, Ultra Low Particulate Air; * Multiple choices were allowed to be selected

Table 5. Approach to SAM and Gynecologic Malignancies Diagnosed during the Pandemic

\begin{tabular}{lc}
\hline Variable & Number (\%) \\
\hline SAM & 23 \\
Postpone after the pandemic & 64 \\
Operate by L/T & 13 \\
Operate by MIS &
\end{tabular}

Do you continue to operate with MIS when SAM turns into OvCa intraoperatively?

Continue staging by L/T

Continue staging by MIS $\quad 19$

Wait for final pathology $\quad 8$

Early Stage OvCa

Postpone after the pandemic 9

Limited staging by L/T (no LND) 3

Full staging by L/T 76

Full staging by MIS

Advanced Stage OvCa

NAC based on laboratory and imaging results 14

NAC after diagnosing by cytology and/or tru-cut biopsy

Diagnostic L/S followed by NAC 7

Diagnostic L/S followed by L/T standard surgery if $\quad 15$ resectable

Diagnostic L/T followed by L/T standard surgery if 20 resectable

Other

Assessment of Advanced Stage OvCa Resectability by MIS

Never performed

Continue to perform

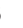

(1)

6

(1)

(1)

15

Endometrioid Type, Grade 1-2, <1/2 Myometrial Invasion EndoCa

Postpone surgery by oral progesterone and/or LR-IUD 10

$\begin{array}{ll}\text { Hysterectomy by L/T } & 12\end{array}$

Hysterectomy by MIS 2

Hysterectomy by MIS and continue based on the FS 19

Hysterectomy by L/T and continue based on the FS 27

Hysterectomy and SLN only by L/T 10

Hysterectomy and SLN only by MIS 10

Full Staging (hysterectomy and PPALND) by L/T 2

Full Staging (hysterectomy and PPALND) by MIS 3

Other 5

High Risk, Grade 3, Deep Myometrial Invasion EndoCa

Postpone surgery 2

Postpone surgery by oral progesterone and/or LR-IUD 2

Decide to postpone or perform the surgery based on 4

the extrauterine spread by imaging

Hysterectomy by L/T 4

Hysterectomy by MIS 1

Hysterectomy and SLN only by L/T 2

Hysterectomy and SLN only by MIS 7

Full Staging (hysterectomy and PPALND) by L/T 62

Full Staging (hysterectomy and PPALND) by MIS 13

Other 3 
Table 5. Continued

\begin{tabular}{|c|c|}
\hline Variable & Number $(\%)$ \\
\hline \multicolumn{2}{|l|}{ Approach to SLN biopsy in EndoCa } \\
\hline Not perform it in routine practice & 51 \\
\hline Performed it before but not during pandemic & 10 \\
\hline SLN only for all cases & 15 \\
\hline Only in low-risk patients for lymphatic metastasis & 6 \\
\hline Only in high-risk patients for lymphatic metastasis & 2 \\
\hline First it for all and PLND & 16 \\
\hline \multicolumn{2}{|l|}{$<2 \mathrm{~cm} \mathrm{CxCa}$} \\
\hline Postpone surgery & 5 \\
\hline $\begin{array}{l}\text { Postpone surgery after conization with negative } \\
\text { surgical margins }\end{array}$ & 3 \\
\hline $\begin{array}{l}\text { Simple hysterectomy by } \mathrm{L} / \mathrm{T} \text { and assessment of } \\
\text { lymph nodes }\end{array}$ & 19 \\
\hline $\begin{array}{l}\text { Radical hysterectomy by } \mathrm{L} / \mathrm{T} \text { and assessment of } \\
\text { lymph nodes }\end{array}$ & 63 \\
\hline $\begin{array}{l}\text { Simple hysterectomy by MIS and assessment of } \\
\text { lymph nodes }\end{array}$ & 1 \\
\hline $\begin{array}{l}\text { Radical hysterectomy by MIS and assessment of } \\
\text { lymph nodes }\end{array}$ & 5 \\
\hline Other & 4 \\
\hline \multicolumn{2}{|l|}{ Approach to SLN biopsy in $<2 \mathrm{~cm} \mathrm{CxCa}$} \\
\hline Not perform it in routine practice & 62 \\
\hline Performed SLN before but not during the pandemic & 5 \\
\hline SLN only for all cases & 11 \\
\hline First SLN and then PLND & 21 \\
\hline $\begin{array}{l}\text { Did not perform SLN before but SLN only for all } \\
\text { cases during the pandemic }\end{array}$ & 1 \\
\hline \multicolumn{2}{|c|}{$\begin{array}{l}\mathrm{CxCa} \text {, Cervical Cancer; EndoCa, Endometrial Cancer; FS, frozen } \\
\text { section; LR-IUD, levonorgestrel-releasing intrauterine system; LND, } \\
\text { lymph node dissection; L/S, laparoscopy; L/T, laparotomy; MIS, } \\
\text { minimally invasive surgery; NAC, Neoadjuvant chemotherapy; OvCa, } \\
\text { ovarian cancer; PLND, lymphadenectomy; PPALND, pelvic and para- } \\
\text { aortic lymphadenectomy; SAM, suspected adnexal mass; SLN, sentinel } \\
\text { lymph node }\end{array}$} \\
\hline
\end{tabular}

staging surgery, a fifth (19\%) continued to L/S. For the management of the cases with early-stage ovarian cancer (ESOC), similarly, three-quarters chose open approach, and nearly one-tenth of the participants preferred to perform MIS (Table 5).

Regarding the patients with advanced-stage ovarian cancer (ASOV), one-fifth of the participants preferred to perform an explorative laparotomy for ASOV, whilst 15 $\%$ preferred diagnostic laparoscopy to triage the patients for either NACT or cytoreductive surgery. Nearly half of the participants $(41 \%)$ chose to have cytology by paracentesis or tru-cut biopsy after which the patient was referred to neo-adjuvant chemotherapy (NACT) without further debulking surgery.

Regarding the management of early-stage endometrial cancer with low-risk features (Grade1 or 2, without deep myometrial invasion), a quarter of the participants performed laparotomy and sent the uterus for frozen section analysis. A fifth of the participants preferred to perform L/S hysterectomy and to proceed with the surgery according to the findings of frozen section analysis. For the patients diagnosed preoperatively with endometrial cancer with high-intermediate risk factors (Grade 3 or deep myometrial invasion), more than half of participants preferred complete staging with laparotomy, but only a tenth of participants performed complete staging by L/S. Regarding the use of SLN biopsy concept in patients with endometrial cancer, more than half of the participants expressed that they did not use this concept while $15 \%$ of the gynecologic oncologists expressed that they routinely used SLN-only concept.

When asked about their attitudes towards cervical cancer, measuring less than $2 \mathrm{~cm}$, approximately two-thirds of the participants expressed that they would perform radical hysterectomy with laparotomy. One-fifth of gynecologic oncologists stated that they preferred simple hysterectomy with an open approach. Only five percent of the participants preferred radical hysterectomy with MIS. When their thoughts about SLN concept was asked for the same circumstance, two-thirds of the participants stressed that they did not use SLN, and more than one-fifth of gynecologic oncologists stated that they performed SLN followed by full pelvic lymphadenectomy. Interestingly, about ten percent of the participants performed only-SLN concept.

The participants were grouped with respect to age (younger than 40 years of age or older), type of hospital (university setting or state hospital), experience in laparoscopic surgery (less than ten years or more), experience in laparoscopic oncologic surgery (less than five years or more), or the COVID status of the hospital (cold or hot designated). The responses were similar between subgroups except that more participants in the older group preferred LEEP or simple hysterectomy rather than a radical hysterectomy for patients with cervical cancer smaller than $2 \mathrm{~cm}$. Gynecologic oncologists with $>10$ years L/S experience used MIS more for SAM. Furthermore, experienced surgeons used L/S more for endometrial cancer patients with both low and highrisk features. Likewise, experienced surgeons preferred simple hysterectomy or LEEP more for a small cervical cancer case (less than $2 \mathrm{~cm}$ ) rather than radical hysterectomy. In busy COVID hospitals, more participants preferred laparotomy over L/S. Moreover, they did not choose diagnostic L/S for evaluating resectability in patients with ASOC. Furthermore, participants in busy facilities selected to use laparotomy more compared to L/S for patients with low or high-risk endometrial cancer. Regarding the use of the SLN concept, more participants in busy settings did not use this approach for patients with endometrial cancer.

\section{Discussion}

To the best of our knowledge, this is the first national survey investigating the gynecologic oncologists' attitudes towards the MIS for the management of the gynecologic malignancies during the COVID-19 pandemic. Our study revealed that the use of MIS declined when compared to the pre-pandemic era. The majority of the participants adopted specific precautions for MIS. Significant number of participants expressed their concerns about the possibility of getting infected. Educational activities and scientific meetings were moved to online platforms. One participant (1\%) was diagnosed 
with SARS-CoV-2infection. The rate of SARS-CoV-2 infection among patients with gynecologic malignancies was $2.7 \%$, with $0.5 \%$ rate of mortality. The majority of the participants stated that in case of a SAM or confirmed ESOC, laparotomy was the preferred technique. Even in ASOC cases, laparotomy or nonsurgical diagnostic approaches such as paracentesis outweighed diagnostic L/S. For early-stage endometrial cancer cases with either low or high intermediate-risk patients, laparotomy was the preferred approach. Moreover, radical hysterectomy with laparotomy outweighed simple hysterectomy or conization for cervical cancer $<2 \mathrm{~cm}$. SLN use either for endometrial or cervical cancer was not the preferred approach. The status of the hospital influenced gynecologic oncologist decision on the surgical approach, so that, participants in busy hospitals mainly used laparotomy, but not the SLN concept. Moreover, age and experience of the surgeon affected the route of surgery. Older and more experienced gynecologic oncologists preferred MIS both for SAM and staging purposes in patients with endometrial cancer.

After the emergence and dramatic increase of the COVID-19 cases worldwide, including Turkey, gynecologic cancer care had to change significantly(Soreide et al., 2020). International and national societies recommended postponing elective surgeries and tailoring the treatment of gynecologic cancers in line with the severity of the pandemic (Uwins et al., 2020). The most striking effect of these recommendations was the sharp decline in the number of gynecologic oncology surgeries, including MIS procedures. Moreover, several anecdotal reports regarding worse post-operative outcomes of asymptomatic COVID-19 patients and demonstration of high virus load in the peritoneal cavity and also in the smoke of energy devices further decreased the number of MIS for the surgical management of the gynecologic cancers (Coccolini F, 2020; Givi et al., 2020; Lei et al., 2020). On the contrary, the use of L/S midst of the COVID-19 pandemic was reported to be safe in the majority of studies(de Santiago et al., 2020; Mintz et al., 2020). In fact, faster post-operative recovery and the advantage of the same day discharge make L/S an attractive option compared to laparotomy (Kornblith et al., 2009; Walker et al., 2009). Despite these convincing data, the participants in our study refrained from practicing MIS in their daily routine. Interestingly, working in "hot" COVID-19 hospitals and lack of experience in MIS were the factors associated with decreased use of MIS during the pandemic. One explanation could be increased operation time for MIS in the hands of inexperienced laparoscopists, which could direct these participants to open surgery. These relatively "inexperienced" participants might feel more comfortable and might complete surgery faster with laparotomy. Evidently, the total number of surgeries declined significantly due to the postponing elective benign surgeries, but there is no data regarding the change in the rate of MIS either for benign or malignant cases during the pandemic.

Recently, outcomes of 126 gynecologic oncologic surgeries from Madrid, Spain, one of the most profoundly affected countries, was reported (de Santiago et al., 2020). Only four patients underwent laparotomy, and the rest of the abdominal surgeries (excluding vulva and breast) was carried out with $\mathrm{L} / \mathrm{S}$. Important to note that these surgeries were performed in a relatively COVID-19-free reference hospital. The authors expressed that MIS for gynecologic cancers was the preferred approach with the advantage of early discharge. On the other hand, a recent study from Turkey reported the outcomes of 200 patients undergoing gynecologic oncology surgery up to 25 May 2020 during the "hot" period of the pandemic (Dursun et al., 2020). Nearly half of the patients had the diagnosis of endometrial carcinoma, where another quarter of the patients were operated for ovarian cancer. Laparotomy was performed in the majority of the cases ( $80 \%$ ). The surgeons reported that they had to modify the operation radicality for ten percent of the cases because of the pandemic. Two patients (1\%) were diagnosed with respiratory distress post-operatively by thorax CT scan with similar findings to COVID-19. However, multiple nasopharyngeal PCR swabs for these two patients failed to confirm SARS-CoV-2 positive results. The findings in this study are in parallel with our results by also confirming the decreased use of L/S among gynecologic oncologists practicing in Turkey.

A global survey conducted via social media showed that one-third of the participants did not continue to perform L/S during the pandemic, whereas the rest of the respondents stated that they still performed MIS with or without modifications of their technique (Martinelli and Garbi, 2020). Interestingly, majority of the participants considered SLN as a reliable tool for nodal evaluation. On the contrary, our study revealed that a significant number of surgeons, mainly working in hot COVID-19 hospitals did not perform SLN in their practice during the pandemic. SLN biopsy is the recommended approach, particularly for endometrial and cervical cancer patients decreasing total operation time and morbidity related to systemic lymphadenectomy (Dogan et al., 2019; Altin et al., 2020). However, because of the issues such as long learning curve, need for experience, and logistic problems (availability of tracer), the use of and preference for the SLN concept might decrease during the pandemic (Freudenberg et al., 2020).As seen from different studies, the preferences of the route of the surgery (laparotomy or laparoscopy), the use of the SLN concept depended on geographic region, the status of the hospital, the experience of the surgeon (Akladios et al., 2020; de Santiago et al., 2020; Dursun et al., 2020).

In our study, the majority of the participants preferred to perform surgery for early-stage gynecologic cancers (including pelvic masses) with either laparotomy or L/S during the pandemic. Postponing surgery with alternative treatment modalities, such as expectant managementof pelvic masses or progestins for early-stage endometrial cancer, was not popular among Turkish gynecologic oncologists. Similarly, in other reports majority of the participants preferred surgery in case of endometrial cancer (de Santiago et al., 2020; Martinelli and Garbi, 2020). On the contrary, this was not the case for patients with ASOC, where after establishing tissue diagnosis, NACT was preferred. This might be related to limited number of intensive care unit (ICU) beds, avoidance from long 
hospitalization after extensive surgery, and reduced blood product reserves. Interestingly, some of these practices, such as surgery for SAM or early endometrial cancer, contradicted the current recommendations of international or national societies (de Santiago et al., 2020; Dursun et al., 2020). Nearly all of these society guidelines and recommendations are based on expert opinions without level I evidence, and real-world data is still limited. We believe that this might be one of the main reasons for nonadherence to the guideline recommendations.

The main strength of our study is the homogeneity of the participants. Members of MİJOD consists of gynecologic oncologists and gynecologists with a special interest in gynecologic oncology. They all have a formal education and experience in minimally invasive gynecologic oncology surgery. Hence, these factors enabled the study group homogenous and representative of the real world. Moreover, the questions of our survey covered a wide range of surgical procedures and different scenarios, which could give a broad insight into the daily routine practices during the pandemic. The anonymity also contributes to more liberal and honest responses without any bias. Our study also has several limitations. The first one is that the data were obtained from a single country. Thus, its generalizability needs to be investigated with further international studies. Moreover, this was a crosssectional study without any follow-up. A second survey after specific time period could have been useful in order to demonstrate changes in practice patterns but in this time, anonymity could have been violated.

In conclusion, the use of MIS in gynecologic oncology decreased during the pandemic in Turkey. More experienced surgeons continued to perform MIS. Surgical treatment was the preferred approach for SAM, early-stage endometrial cancer, and ESOC. However, NACT was more popular compared to radical upfront surgery.

\section{Author Contribution Statement}

NUD designed the study, performed the statistical analysis, wrote the manuscript, revised it intellectually, ST designed the questionnaire, revised the manuscript intellectually, DV designed the questionnaire, collected the data, revised the manuscript intellectually, EB designed the questionnaire, collected the data, revised the manuscript intellectually, SD designed the questionnaire, collected the data, revised the manuscript intellectually, $\mathrm{HC}$ collected the data, assisted the statistical analysis, revised the manuscript intellectually, FO collected the data, revised the manuscript intellectually $\mathrm{MG}$ designed the questionnaire, collected data, revised the manuscript intellectually, CT designed the study, designed the questionnaire, performed the statistical analysis, wrote the manuscript, revised it intellectually.

\section{Acknowledgments}

There is no grant for this research from any funding agency. The study was approved by both Turkish Ministry of Health (2020-06-03T09-58-03) and Koc University Institutional Review Board (2020.260.IRB3.101). The authors declare no conflict of interest.

\author{
Informed consent \\ Informed consent was obtained from the participants \\ online.
}

\section{Conflict of Interest}

The authors declared no conflict of interest.

\section{References}

Akladios C, Azais H, Ballester M, et al (2020). Recommendations for the surgical management of gynecological cancers during the COVID-19 pandemic - FRANCOGYN group for the CNGOF. J Gynecol Obstet Hum Reprod, 49, 101729.

Altin D, Taskin S, Kahramanoglu I, et al (2020). Combination of sentinel lymph node mapping and uterine frozen section examination to reduce side-specific lymphadenectomy rate in endometrial cancer: a Turkish Gynecologic Oncology Group study (TRSGO-SLN-002). Int J Gynecol Cancer, 30, 1005-11.

Coccolini F TD, Puglisi A, Giordana C, et al (2020). SARS-CoV-2 is present inperitoneal fuid in COVID-19 patients. Ann Surg, 272, 240-2.

Cucinotta D, Vanelli M (2020). WHO Declares COVID-19 a Pandemic. Acta Biomed, 91, 157-60.

de Santiago J, Yelo C, M FC, et al (2020). COVID-19: gynecologic cancer surgery at a single center in Madrid. Int J Gynecol Cancer, 30, 1108-12.

Demirbilek Y, Pehlivanturk G, Ozguler ZO, et al (2020). COVID-19 outbreak control, example of ministry of health of Turkey. Turk J Med Sci, 50, 489-94.

Di Saverio S, Khan M, Pata F, et al (2020). Laparoscopy at all costs? Not now during COVID-19 outbreak and not for acute care surgery and emergency colorectal surgery: A practical algorithm from a hub tertiary teaching hospital in Northern Lombardy, Italy. J Trauma Acute Care Surg, 88, 715-8.

Dogan NU, Dogan S, Favero G, et al (2019). The basics of sentinel lymph node biopsy: Anatomical and Pathophysiological Considerations and Clinical Aspects. J Oncol, 2019, 3415630.

Dursun P, Dervisoglu H, Daggez M, et al (2020). Performing gynecologic cancer surgery during the COVID-19 pandemic in Turkey: A multicenter retrospective observational study. Int J Gynaecol Obstet, 2020.

Fader AN, Huh WK, Kesterson J, et al (2020). When to operate, hesitate and reintegrate: Society of Gynecologic Oncology Surgical Considerations during the COVID-19 Pandemic. Gynecol Oncol, 158, 236-43.

Freudenberg LS, Paez D, Giammarile F, et al (2020). Global impact of COVID-19 on nuclear medicine departments: An International Survey in April 2020. J Nucl Med, 61, 1278-83.

Givi B, SchiffBA, Chinn SB, et al (2020). Safety recommendations for evaluation and surgery of the head and neck during the COVID-19 pandemic. JAMA Otolaryngol Head Neck Surg, 146, 579-84.

Kornblith AB, Huang HQ, Walker JL, et al (2009). Quality of life of patients with endometrial cancer undergoing laparoscopic international federation of gynecology and obstetrics staging compared with laparotomy: a Gynecologic Oncology Group study. J Clin Oncol, 27, 5337-42.

Lei S, Jiang F, Su W, et al (2020). Clinical characteristics and outcomes of patients undergoing surgeries during the incubation period of COVID-19 infection. E-Clin Med, 21, 100331.

Martinelli F, Garbi A (2020). Change in practice in gynecologic 
oncology during the COVID-19 pandemic: a social media survey. Int J Gynecol Cancer, 30, 1101-7.

(MİJOD) TSoGOaTEP ( 2020). (MIJOD) TSoGOaTEP (2020). Important aspects of minimal invasive gynecological surgery during covid-19 pandemic.

Mintz Y, Arezzo A, Boni L, et al (2020). The risk of COVID-19 transmission by laparoscopic smoke may be lower than for laparotomy: a narrative review. Surg Endosc, 34, 3298-305.

Nakayama J, El-Nashar SA, Waggoner S, et al (2020). Adjusting to the new reality: Evaluation of early practice pattern adaptations to the COVID-19 pandemic. Gynecol Oncol, 158, 256-61.

Oncology TSoG (2020). Management of gynecologic cancers during the COVID-19 pandemic. Med J Aegean Clin, 2020.

Rusch VW, Wexner SD, American College of Surgeons Covid-19 Communications Committee BoR, et al (2020). The American College of Surgeons Responds to COVID-19. $J$ Am Coll Surg, 231, 490-6.

Soreide K, Hallet J, Matthews JB, et al (2020). Immediate and long-term impact of the COVID-19 pandemic on delivery of surgical services. Br J Surg, 2020.

Spinelli A, Pellino G (2020). COVID-19 pandemic: perspectives on an unfolding crisis. Br J Surg, 107, 785-7.

Uwins C, Bhandoria GP, Shylasree TS, et al (2020). COVID-19 and gynecological cancer: a review of the published guidelines. Int J Gynecol Cancer, 30, 1424-33.

Walker JL, Piedmonte MR, Spirtos NM, et al (2009). Laparoscopy compared with laparotomy for comprehensive surgical staging of uterine cancer: Gynecologic Oncology Group Study LAP2. J Clin Oncol, 27, 5331-6.

\section{(c) (7) (2)}

This work is licensed under a Creative Commons AttributionNon Commercial 4.0 International License. 